Ann. Génét. Sél. anim., I980, 12 (2), 215-218.

\title{
Note sur l'inverse d'une matrice de parenté
}

\author{
M. POUTOUS \\ Station de Génetique Appliquée, \\ Centre national de Recherches zootechniques, I.N.R.A., \\ 78350 Jouy-en-Josas
}

\begin{abstract}
Résumé
L'inverse de la matrice des variances et covariances génétiques, dans le cas d'additivité, présente une structure particulière. Les seuls éléments non nuls concernent les groupes parentsparents et parents-descendants directs et de ce fait sont en nombre réduit. Les contributions d'un nouvel individu sont de nature additive et ne dépendent que du type d'ascendance retenu et, éventuellement, de la consanguinité moyenne de ses parents.

Les formules générales de calcul sont rappelées et étendues, pour des populations non consanguines, à des pedigrees avec un nombre quelconque d'ascendants directs.
\end{abstract}

\section{Intérêt}

La matrice inverse des variances et covariances génotypiques, dans 1'hypothèse d'additivité est fréquemment requise, en particulier pour le calcul des indices des reproducteurs. Des méthodes générales de résolution numérique étant déjà connues (par ex. réf. 2), l'objectif de cette note est simplement de rappeler, par une voie théorique un peu différente, d'une part que le nombre d'éléments nonnuls de cette matrice est réduit aux seules liaisons parents direct-parents directs et parent directs-descendants directs, d'autre part que la résolution numérique est tout à fait simple dans le cas de non consanguinité et qu'il n'y a alors pas de raison pour ne pas tenir compte des liens de parenté dans le classement des reproducteurs.

\section{Solution générale}

Nous appellerons matrice de parenté (G) le double de la matrice des coeffcients de parenté, au sens de Malécot, pour tous les individus d'une population donnée. Sous l'hypothèse d'additivité, G est égale à la matrice des variances et 
covariances génétiques divisée par la valeur de la variance génétique (par exemple réf. I). Soit une population $A$ pour laquelle on connaît $G$ et $G^{-1}$. Introduisons un nouvel individu $I$, sans descendants dans $A$ et cherchons les nouvelles valeurs $G_{*}$ et $\mathrm{G}_{*}^{-1}$ de $\mathrm{A}$ augmentée de I. L'ensemble des relations de $\mathrm{I}$ avec $\mathrm{A}$ peut se schématiser de la façon suivante, les flèches indiquant les sens de descendance possibles,

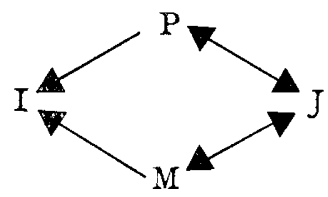

I e coefficient de parenté de I avec J est alors égal à la moyenne des coefficients de parenté $q$ et $r$ de $\mathrm{J}$ avec $\mathrm{P}$ et $\mathrm{M}$. Appelons $f_{x}$ le coefficient de consanguinité d'un individu $\mathrm{X} . \mathrm{G}_{*}$ est égale à $\mathrm{G}$ augmentée de la ligne et de la colonne correspondant à I. On a, les lettres entre parenthèses symbolisant 1'animal pour la ligne correspondante :

$$
\mathrm{G}_{*}=\left[\begin{array}{rrrr}
\mathrm{I}+f_{\mathrm{I}} & \frac{\mathrm{I}+f_{\mathrm{P}}}{2}+f_{\mathrm{I}} & \frac{\mathrm{I}+f_{\mathrm{MI}}}{2}+f_{\mathrm{I}} & q+r \\
\frac{\mathrm{I}+f_{\mathrm{P}}}{2}+f_{\mathrm{I}} & \mathrm{I}+f_{\mathrm{P}} & 2 f_{\mathrm{I}} & 2 q \\
\frac{\mathrm{I}+f_{\mathrm{M}}}{2}+f_{\mathrm{I}} & 2 f_{\mathrm{I}} & \mathrm{I}+f_{\mathrm{M}} & 2 r \\
q+r & 2 q & 2 r & \mathrm{I}+f_{\mathrm{J}}
\end{array}\right](\mathrm{I})
$$

En désignant par le symbole $(=)$, les valeurs correspondantes de $\mathrm{G}^{-1}$, on vérifie que $\mathrm{G}_{*}^{-1} \times \mathrm{G}_{*}=\mathrm{I}$, matrice unitaire, si on donne à $\mathrm{G}_{*}^{-1}$ les valeurs suivantes :

$$
\mathrm{G}_{*}^{-1}\left[\begin{array}{cccc}
+\Delta & -\frac{\Delta}{2} & -\frac{\Delta}{2} & 0 \\
-\frac{\Delta}{2} & (=)+\frac{\Delta}{4} & (=)+\frac{\Delta}{4} & (=) \\
-\frac{\Delta}{2} & (=)+\frac{\Delta}{4} & (=)+\frac{\Delta}{4} & (=) \\
0 & (=) & (=) & (\mathrm{I})
\end{array}\right](\mathrm{I})
$$

Avec $\Delta=-\frac{2}{\mathrm{I}-\frac{f_{\mathrm{M}}+f_{\mathrm{P}}}{2}}$

Cette règle peut être appliquée successivement, d'abord aux fondateurs en admettant qu'ils ne sont pas apparentés, puis à leurs descendants directs puis aux descendants directs de ces derniers, etc... On obtient alors la matrice inverse de la population et on vérifie que ses seuls éléments non-nuls sont bien ceux qui ont trait aux relations : parents directs entre eux et avec leurs descendants directs. 


\section{Remarque:}

Seule la consanguinité des parents intervient dans la valeur de $\Delta$ et, sauf pour certaines populations expérimentales, avec un poids peu important.

\section{Cas des populations non consanguines}

En pratique, les éleveurs évitent d'accoupler des animaux proches parents et la plupart de nos races domestiques sont à larges effectifs et ouvertes, par conséquent les coefficients de consanguinité sont faibles. Par ailleurs, au moins pour les grandes espèces à intervalle de génération long, les pedigree disponibles ne comprennent que peu de générations d'ascendants. Aussi peut-on souvent négliger la consanguinité, qu'elle soit réellement faible ou qu'on ne puisse pas la calculer.

Une modification de la notion de pedigree, de peu d'intérêt dans le cas général, peut être également introduite, en fonction de considérations autres que les seules liaisons génétiques. Nous admettrons donc qu'un individu I peut avoir $n$ ascendants directs, sous la réserve que ceux-ci soient les premiers sur la ligne choisie. Par exemple on peut concevoir des pedigrees avec ascendance mâle seulement et dans ce cas les ascendants directs seront : le père, le père de la mère, le père de la mère de la mère, etc... Le raisonnement qui suit n'est valable que si les liens de parenté sont calculés seulement à partir des ascendants directs. Par exemple, si on retient le père et le grand-père maternel, deux demi-frères de mère seront considérés comme ayant seulement le même grand-père. Cette approximation doit être justifiée pour chaque application.

Nous garderons le même symbolisme que dans le cas général, les $n$ ancêtres directs de I étant symbolisés par 2 quelconques d'entre eus $\mathrm{P}$ et $\mathrm{M}$ ( $p$ et $m$ désignant les coefficients de parenté de $\mathrm{I}$ avec $\mathrm{P}$ et $\mathrm{M}$ ) et $\mathrm{J}$ un apparenté quelconque de I par l'intermédiaire d'un ancêtre direct de I. L,a solution pour $\mathrm{G}_{*}^{-1}$ s'écrit :

$$
\mathrm{G}_{*}^{-1}=\left[\begin{array}{lllc}
-\Delta 2 m & (=)+\Delta 2 p 2 m & (=)+\Delta(2 m)^{2} & (=) \\
+\Delta & -\Delta 2 p & -\Delta 2 m & 0 \\
-\Delta 2 p & (=)+\Delta(2 p)^{2} & (=)+\Delta 2 p 2 m & (=) \\
0 & (=) & (=) & (=)
\end{array}\right]
$$

Avec : $\Delta=\frac{\mathrm{I}}{\mathrm{I}-4^{\Sigma}}$

$\Sigma$ étant la somme des carrés des coefficients de parenté de I avec chacun de ses ancêtres directs.

\section{Gas particuliers}

- o ancêtres directs :

$$
p=m=0, \Delta=\mathrm{r}
$$

- père (les solutions seraient identiques pour la mère)

$$
\begin{aligned}
& p=\frac{\mathrm{I}}{4}, m=0, \\
& \Delta=\frac{4}{3}
\end{aligned}
$$


- père et mère :

$p=m=\frac{\mathrm{I}}{4}, \Delta=2$. On retrouve les formules générales si les parents ne sont pas consanguins.

- père $(\mathrm{P})$ et grand-père maternel $(\mathrm{M})$ :

$$
\begin{aligned}
& p=\frac{\mathrm{I}}{4}, \quad m=\frac{\mathrm{I}}{8}, \\
& \Delta=\frac{\mathrm{I} 6}{\mathrm{II}}
\end{aligned}
$$

Received for publication in august I980.

\section{Summary}

$A$ note upon the inverse of a kindship matrix.

In the case of additivity the inverse matrix of genetic variances and covariances has a peculiar structure. The only non zero elements are in connection with the parents/parents and parents /direct progeny groups and, as a matter of fact, are in a reduced number. The contributions of a new individual are of additive nature and depend only on the type of ancestry which is considered and essentially the average in breeding of its parents. The general computation formulae are recalled and extended for non inbred populations having pedigrees with any number of direct ancestry.

\section{Références bibliographiques}

(1) Gilıors M., r964. La velation d'identité en génétique. Thèse, Fac. Sciences, Paris, 294 p. (2) Henderson, 1976. A simple method for computing the inverse of a numerator relationship matrix used in prediction of breeding values. Biometries, 32, 69-83. 